\title{
O girino de Scinax nebulosus (Amphibia, Anura, Hylidae)
}

\author{
Marcia dos Reis Gomes ${ }^{1}$, Ana Claudia Reis Alves $^{1} \&$ Oswaldo Luiz Peixoto²
}

1. Universidade Federal do Rio de Janeiro, Instituto de Biologia, Departamento de Zoologia, Laboratório de Anfíbios, Caixa Postal 68044, 21941-590 Rio de Janeiro, RJ, Brasil.
(marciadrgomes@gmail.com; anaclaudiareis.alves@gmail.com)
2. Universidade Federal Rural do Rio de Janeiro, Instituto de Biologia, Departamento de Biologia Animal, 23870-000 Seropédica, RJ, Brasil. (pedra10@yahoo.com)

\begin{abstract}
The tadpole of Scinax nebulosus (Amphibia, Anura, Hylidae). The tadpole of Scinax nebulosus (Spix, 1824) is described and illustrated from specimens collected in the state of Pernambuco, Northeastern Brazil. The presence of a labial arm in the oral disc, a diagnostic feature of the tadpole of the Scinax rostratus group, is reported. The previous identification of S. nebulosus tadpoles in the literature is questioned and a comparison with all other known tadpoles of the Scinax rostratus group is provided, based on literature.
\end{abstract}

KEYWORDS. Scinax rostratus group, labial arm, Brazil, Pernambuco.

RESUMO. É descrito e ilustrado o girino de Scinax nebulosus (Spix, 1824), proveniente do estado de Pernambuco, nordeste do Brasil. O girino apresenta braço labial no disco oral. A presença dessa estrutura associa claramente essa espécie ao grupo de Scinax rostratus. A identificação do girino anteriormente atribuído a Scinax nebulosus é considerada errônea e com base na literatura é feita a comparação com os demais girinos conhecidos do grupo.

PALAVRAS-CHAVE. Grupo Scinax rostratus, braço labial, Brasil, Pernambuco.

O grupo de Scinax rostratus, de distribuição predominantemente amazônica, é formado por dez espécies: Scinax boulengeri (Cope, 1887), Scinax constrictus Lima, Bastos \& Giaretta 2004, Scinax garbei (Miranda Ribeiro, 1926), Scinax jolyi Lescure \& Marty, 2001, Scinax kennedyi (Pyburn, 1973), Scinax nebulosus (Spix, 1824), Scinax pedromedinae (Henle, 1991), Scinax proboscidea (Brongersma, 1933), Scinax rostratus (Peters, 1863) e Scinax sugillatus (Duellman, 1973). No Brasil são registradas cinco espécies desse grupo, das quais, segundo Frost (2013), S. nebulosus é assinalada para a Amazônia e o Nordeste; S. constrictus está restrita ao Centro-Oeste (Cerrado); S. garbei e S. pedromedinae são apenas registradas para a Amazônia, onde ocorre, também, segundo Sturaro et al. (2010), S. rostratus. Seis espécies pertencentes ao grupo de Scinax rostratus têm os girinos conhecidos: S. boulengeri, S. garbei, S. rostratus, $S$. sugillatus, $S$. pedromedinae e apenas o atribuído a $S$. nebulosus não apresenta o braço labial assinalado no disco oral. Essa estrutura é considerada uma sinapomorfia para o grupo de Scinax rostratus (McDiarmid \& Altig, 1990, 1999; Duellman \& Wiens, 1992; Faivovich, 2002; Faivovich et al., 2005).

DE SÁ et al. (1997) ao descreverem o girino de $S$. nebulosus de Belém, estado do Pará, Brasil, consideraram, devido à ausência do braço labial na amostra analisada, que a identificação poderia estar equivocada, ou que $S$. nebulosus não pertenceria ao grupo de Scinax rostratus. Os girinos analisados e descritos por DE SÁ et al. (1997) foram assim identificados por M. L. Crump e W. E. Duellman, provavelmente, por serem provenientes da localidadetipo de Hyla egleri (Lutz, 1968), espécie sinonimizada a S. nebulosus por Hoogmoed \& Gruber (1983). Esta não seria a primeira identificação errada de girinos do grupo de Scinax rostratus. Duellman (1970) descreveu um girino do grupo de Scinax ruber como sendo de S. rostratus, o que foi corrigido por McDiarmid \& Altig (1990). Posteriormente, RADA DE MARTinez (1990) descreveu o girino de Dendropsophus minutus como de S. rostratus, o que foi corrigido por Hero \& Mijares-Urrutia (1995).

De acordo com as análises de FaIvovich (2002), dentre as dez sinapomorfias que definem o grupo de Scinax rostratus, quatro se referem ao disco oral das larvas, sendo uma delas a posição da terceira fileira inferior de dentículos na extremidade do braço labial. Em função da ausência do braço labial e da falta de indícios que atribuíssem, com certeza, a larva descrita por De SÁ et al. (1997) à $S$. nebulosus, FAIVOVICH (2002) não incluiu na sua matriz os caracteres larvais dessa espécie. Girinos e adultos de $S$. nebulosus colecionados no estado de Pernambuco, nordeste do Brasil, possibilitaram a correta identificação do girino dessa espécie que é apresentada no presente trabalho.

\section{MATERIAL E MÉTODOS}

Adultos e girinos foram coletados em um açude com cerca de $50 \mathrm{~cm}$ de profundidade nas bordas no município de Cabo de Santo Agostinho, estado de Pernambuco, no período de agosto a setembro de 2001, a metamorfose de um girino possibilitou a sua identificação. $\mathrm{O}$ material está depositado na coleção ZUFRJ do Departamento de Zoologia, Instituto de Biologia, Universidade Federal do Rio de Janeiro (ZUFRJ 8848-8851, adultos; ZUFRJ 8902, girinos e recém metamorfoseado).

Material examinado. Estado de Pernambuco: Cabo de Santo Agostinho, COMPESA, Gurjaú, 29.08 a 
02.09.2001, Ana Carolina Oliveira Queiroz Carnaval col. - ZUFRJ 8902 (três girinos e um recém-metamorfoseado); ZUFRJ 8848-8851 (adultos).

Os girinos foram medidos com ocular milimetrada e desenhados em câmara-clara acoplada a microscópio estereoscópico Leica MZ6. As medidas, seguindo Altig (2007), foram tomadas em mm: comprimento total, comprimento do corpo, largura do corpo, altura do corpo (logo atrás dos olhos), comprimento da cauda, altura máxima da cauda, altura máxima da membrana superior, altura da membrana inferior, focinho-surgimento da cauda, diâmetro do olho, diâmetro da narina, distância interocular, distância internasal, distância do olho à narina, distância da narina ao focinho, distância do olho ao focinho, e largura do disco oral. A nomenclatura das estruturas orais e a fórmula dentária labial seguem o proposto por Altig \& McDiarmid (1999). Os estágios (E) foram determinados de acordo com a tabela de Gosner (1960). A descrição e ilustração do girino são baseadas em um exemplar no estágio 37, enquanto o disco oral é figurado de um girino no estágio 36.

\section{RESULTADOS}

Descrição do girino de Scinax nebulosus. ZUFRJ 8902, estágio 37 (Figs 1-3, Tab. I), medidas do exemplar em mm; comprimento total 24,3; comprimento do corpo 7,2; largura do corpo 4,2; altura do corpo 4,1; altura da cauda 5,1; altura da membrana superior 1,5 ; altura da membrana inferior 1,8; focinho-surgimento da cauda 4,4; diâmetro do olho 1,2; diâmetro da narina 0,2; distância interocular 3,1; distância internasal 2,1; distância do olho à narina 1,1; distância da narina ao focinho 1,5 ; distância do olho ao focinho 2,6; largura do disco oral 1,8. Corpo oval em vista dorsal, mais longo do que largo (largura 58\% do comprimento do corpo), ligeiramente mais largo do que alto (altura 97\% da largura do corpo); altura máxima, em vista lateral, localizada no último terço do corpo. Comprimento do corpo com cerca de 30\% do comprimento total. Em vista dorsal, focinho arredondado. Narinas arredondadas, localizadas dorsalmente, visíveis em vista lateral, ligeiramente mais próximas dos olhos do que do focinho. Distância internasal correspondendo a 68\% da distância interorbital. Olhos laterais com diâmetro correspondendo a $29 \%$ da altura do corpo, distância interorbital 74\% da largura do corpo e 2,6 vezes maior do que o diâmetro do olho. Espiráculo sinistro, com a parede interna livre do corpo, abertura voltada posteriormente, localizado abaixo da linha média. Tubo cloacal mediano, com ambas as paredes ligadas diretamente à nadadeira ventral, com abertura voltada posteriormente (Fig. 6). Cauda levemente arqueada, mais alta do que o corpo, afilada na extremidade. Membrana inferior ligeiramente mais alta do que a membrana superior.

Disco oral anteroventral, com largura equivalendo a $43 \%$ da largura do corpo; uma fileira de papilas marginais com larga interrupção anterior e interrupção ventral um pouco mais estreita; papilas marginais próximas ao braço labial mais alongadas; papilas submarginais laterais pequenas e em número reduzido. Fórmula das fileiras de dentículos labiais 2(2)/3(1); braço labial (Fig. 7) com 6 dentículos. Bico córneo robusto e serrilhado com uma pequena projeção mediana na maxila.

Coloração (Fig. 5): Em vida, girino sem pigmentação marcante, com aspecto geral transparente, mostrando uma nuance olivácea em vista dorsal; em vista lateral, evidenciase uma estreita faixa enegrecida do focinho aos olhos, que são discretamente acobreados; ventre esbranquiçado. Em formol a 5\%, musculatura caudal e corpo pardo-claro, em vista lateral, com uma faixa marrom dos olhos ao disco oral, e pequenas manchas, de igual colorido, sob os olhos; ventre transparente e olhos enegrecidos.

\section{DISCUSSÃO}

Os girinos descritos de Scinax boulengeri (LÉON, 1969; Duellman, 1970), S. garbei (Duellman, 1978), S. rostratus (Hero \& Mijares-Urrutia, 1995; figurado por LyNCH, 2006), S. sugillatus (McDiarmid \& Altig, 1990) e S. pedromedinae (Duellman, 2005) apresentam braço labial no disco oral, como o girino aqui descrito. Em relação a esses girinos, o de $S$. nebulosus distinguese por apresentar: tamanho menor (CT $=24,3 \mathrm{~mm}$; E37), S. garbei (CT = $29 \mathrm{~mm}$; E36), S. rostratus $(\mathrm{CT}=39,2$ $\mathrm{mm}$; E39); narina um pouco mais perto dos olhos do que do focinho, equidistante em $S$. garbei e mais próxima do focinho do que dos olhos em S. rostratus; o disco oral posicionado anteroventralmente, terminal em S. rostratus; primeira fileira inferior de dentículos labiais interrompida, 2(2)/3(1), não interrompida em S. garbei, 2(2)/3; maxila com projeção mediana, ausente em S. garbei e S. rostratus; papilas submarginais em número reduzido, ausentes em $S$. garbei, numerosas em S. rostratus; ventre esbranquiçado

Tab. I. Medidas, em mm, dos girinos de Scinax nebulosus (Spix, 1824) (ZUFRJ 8902).

\begin{tabular}{lccc}
\hline & Estágio 37 & Estágio 36 & Estágio 28 \\
\hline Comprimento total & 24,3 & 23 & 17,3 \\
Comprimento do corpo & 7,2 & 6,9 & 5,3 \\
Focinho-surgimento da cauda & 4,4 & 4,4 & 3,6 \\
Altura do corpo & 4,1 & 3,3 & 2,7 \\
Altura da cauda & 5,1 & 4,3 & 3,5 \\
Altura da membrana superior & 1,5 & 1,3 & 1,0 \\
Altura da membrana inferior & 1,8 & 1,5 & 1,2 \\
Diâmetro do olho & 1,2 & 1,2 & 0,8 \\
Diâmetro da narina & 0,2 & 0,3 & 0,2 \\
Largura do corpo & 4,2 & 3,9 & 3,0 \\
Distância interocular & 3,1 & 2,9 & 2,1 \\
Distância internasal & 2,1 & 2,0 & 1,7 \\
Distância narina-focinho & 1,5 & 1,2 & 1,1 \\
Distância olho-focinho & 2,6 & 2,4 & 2,0 \\
Largura do disco oral & 1,8 & 1,8 & 1,4 \\
Distância olho-narina & 1,1 & 1,0 & 0,8 \\
Comprimento da cauda & 17,1 & 16,1 & 12,0 \\
\hline
\end{tabular}


1

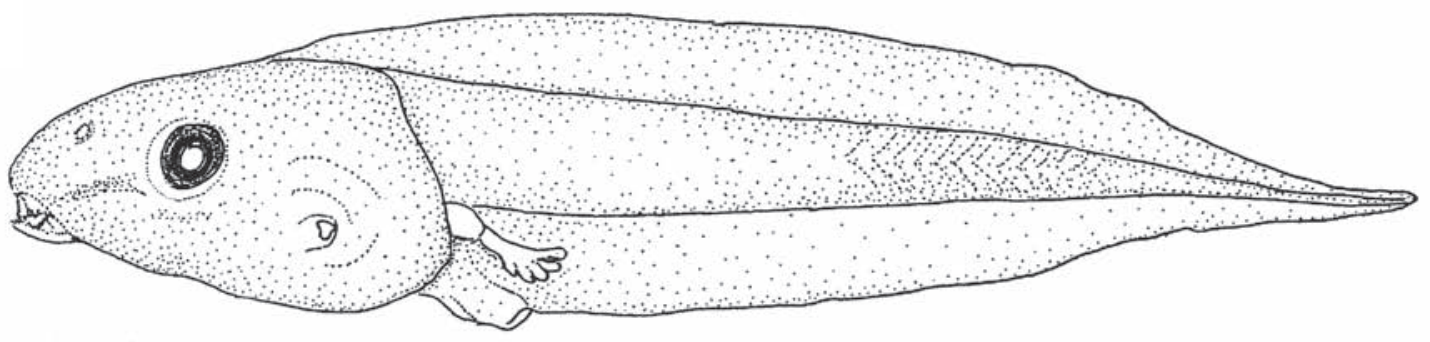

2

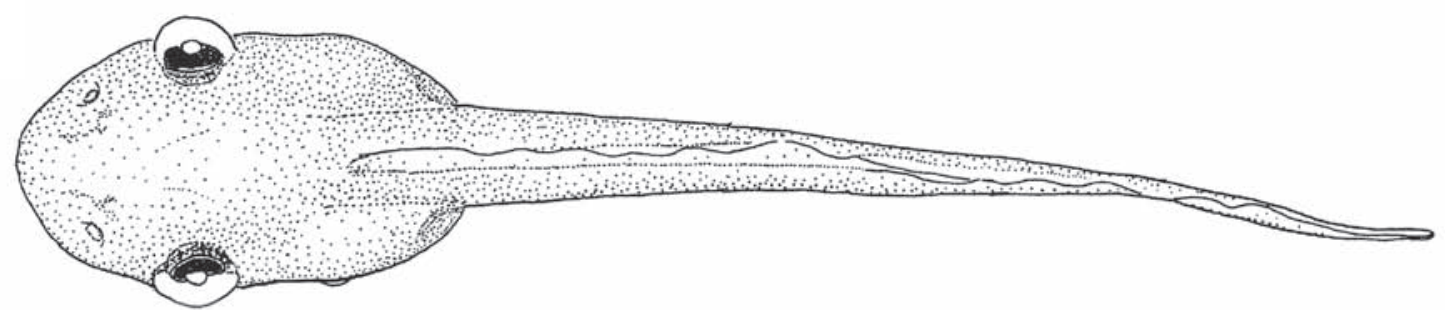

3

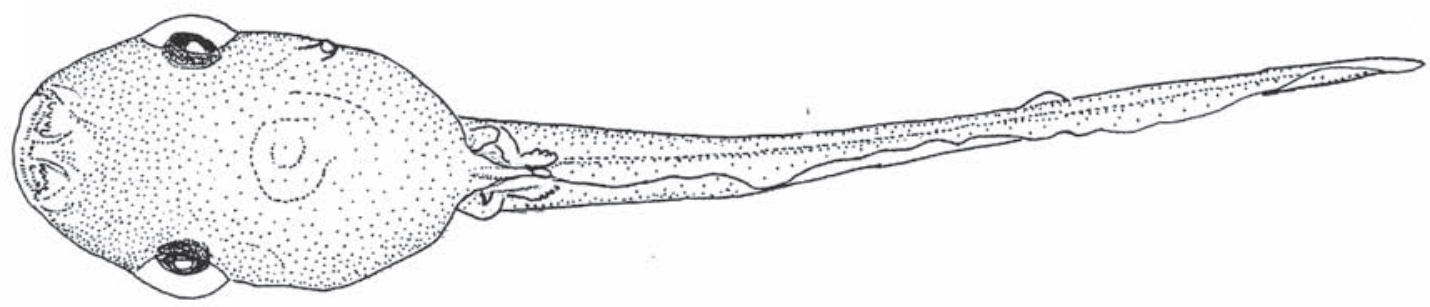

\section{$2 \mathrm{~mm}$}

Figs 1-3. Girino de Scinax nebulosus (Spix, 1824) (ZUFRJ 8902, estágio 37): 1, vista lateral; 2, vista dorsal; 3, vista ventral.

e corpo transparente, ventre transparente e corpo opaco em $S$. rostratus; corpo ligeiramente mais largo do que alto, mais alto do que largo em $S$. rostratus. O girino de S. pedromedinae foi descrito por Duellman (2005) no estágio 25, destacando o autor o seu pequeno tamanho, e distingue-se por apresentar narinas equidistantes dos olhos e do focinho (levemente mais próximas do olho em $S$. nebulosus); disco oral mais amplo (66\% da largura do corpo; 43\% em S. nebulosus); tubo anal destro, livre da membrana ventral (mediano e aderido em S. nebulosus); disco oral sem papilas submarginais (poucas em S. nebulosus), o braço labial não apresenta fileiras de dentículos, o que foi atribuído ao estágio imaturo dos girinos.

O girino de $S$. nebulosus difere de $S$. sugillatus e de $S$. boulengeri por ser menor (35,4 mm; E37 e 33,5 mm; E38, respectivamente), ter o corpo ligeiramente mais largo do que alto, enquanto o das outras espécies tem o corpo mais alto do que largo. As narinas em $S$. nebulosus estão mais próximas dos olhos do que do focinho e em $S$. sugillatus estão mais próximas do focinho. Em relação ao disco oral, S. nebulosus apresenta um número reduzido de papilas submarginais (Fig. 4), enquanto S. sugillatus não possui papilas submarginais, enquanto que $S$. boulengeri apresenta. Na fórmula de dentículos labiais difere de $S$. sugillatus que tem a primeira e a segunda fileiras superiores interrompidas,

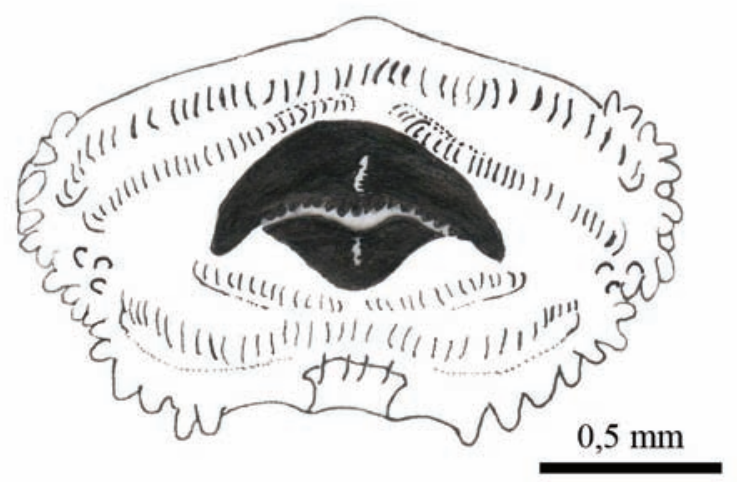

Fig. 4. Disco oral de Scinax nebulosus (Spix, 1824) (ZUFRJ 8902, estágio 36).

2(1-2)/3(1) e de S. boulengeri que não apresenta interrupção na primeira fileira inferior de dentículos labiais, 2(2)/3. A terceira fileira inferior de dentículos labiais de $S$. nebulosus tem de 4 a 6 dentículos labiais (Fig. 4), enquanto que em S. sugillatus, a terceira fileira de dentículos labiais tem de 2 a 6 dentículos e em $S$. boulengeri a terceira fileira tem de 5 a 10 dentículos. A maxila em $S$. nebulosus apresenta uma projeção mediana, ausente em $S$. sugillatus e em $S$. boulengeri. 


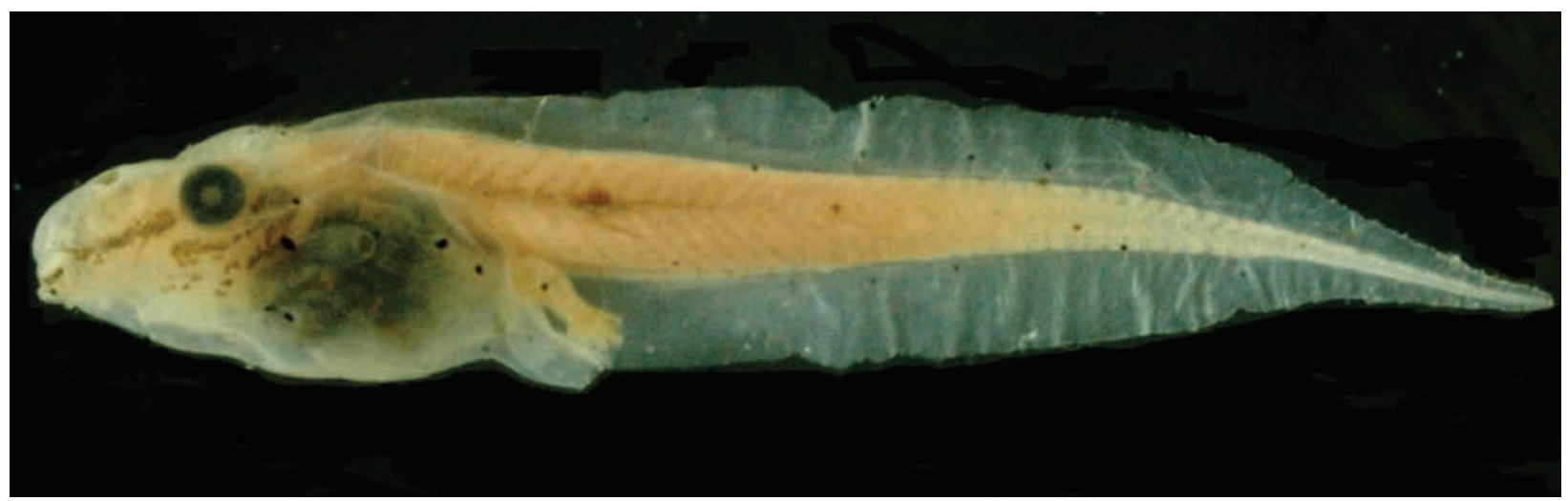

Fig. 5. Girino de Scinax nebulosus (Spix, 1824) em vista lateral (ZUFRJ 8902, estágio 37).

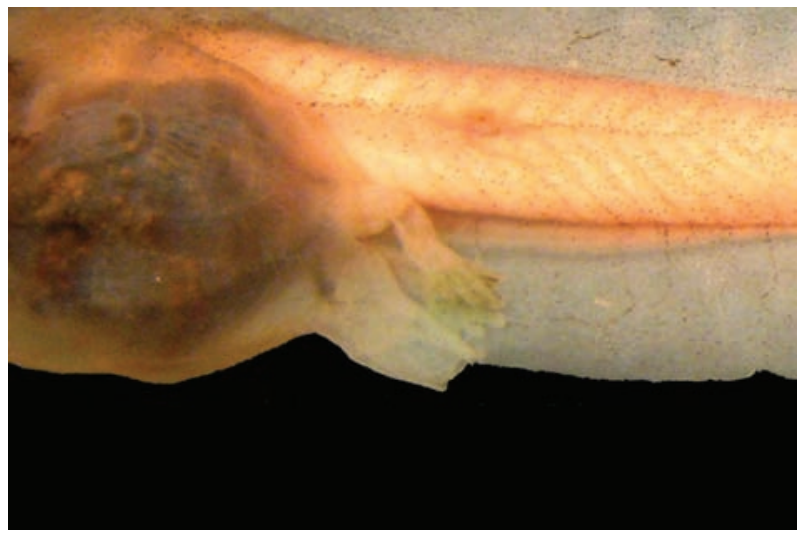

Fig. 6. Girino de Scinax nebulosus (Spix, 1824), detalhe do tubo cloacal e do espiráculo.

O tubo cloacal do girino de S. nebulosus enquadra-se no figurado por Altig \& MCDiARmid (1999, fig. 3.5: H; L) sendo mediano e tendo abertura voltada posteriormente, o que amplia a variação do estado desse caráter no grupo de Scinax rostratus.

Com os dados obtidos, concluímos que houve erro de identificação nos girinos atribuídos anteriormente a $S$. nebulosus, descritos por DE SÁ et al. (1997) e pertencentes ao mesmo lote ilustrado como Ololygon nebulosa por Duellman \& Trueb $(1986,1994)$, reproduzido por Altig \& Johnston (1989: Fig. 7C) e Altig \& McDiarmid (1999: Fig. 12.1C) que não se enquadram como do grupo de $S$. rostratus pela ausência de braço labial, por terem o disco oral margeado por papilas sem interrupção posterior e por apresentarem cauda mais alta do que os demais girinos do grupo; se distinguem daqueles de $S$. nebulosus, ora descritos (1) pelo maior tamanho (52,3 mm contra 24,3 mm, estágio 37); (2) pela forma geral do corpo (mais alto do que largo) e (3) nadadeiras mais altas, assemelhando-se a girinos do clado de Scinax ruber.

Agradecimentos. A Ana Carolina Oliveira Queiroz Carnaval pela coleta do material utilizado na descrição. A W. E. Duellman pelo fornecimento de cópias de trabalhos. A Nedyson Araújo Silva e Cyro de Luna Dias Neto pela ajuda com a digitalização das figuras e a Joice Ruggeri pela obtenção de bibliografia.

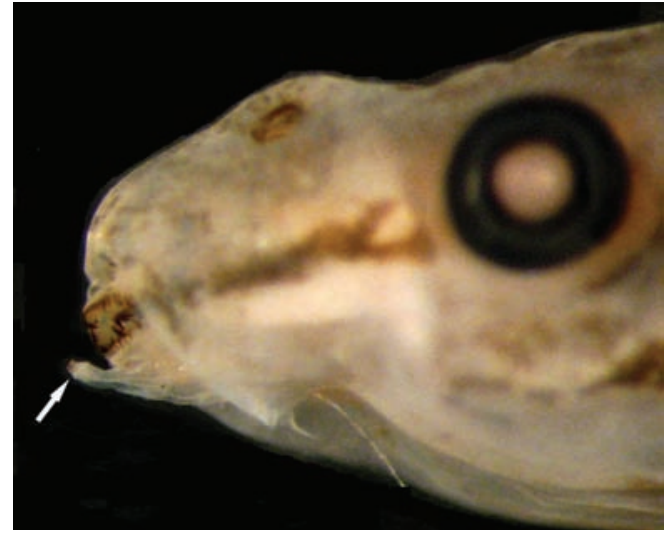

Fig. 7. Girino de Scinax nebulosus (Spix, 1824), destaque para o braço labial.

\section{REFERÊNCIAS BIBLIOGRÁFICAS}

Altig, R. 2007. A primer for the morphology of Anuran tadpoles. Herpetological Conservation and Biology 2(1):71-74.

Altig, R. \& Johnston, G. F. 1989. Guilds of anuran larvae: Relationships among developmental modes, morphologies, and habitats. Herpetological Monographs 3:81-109.

Altig, R. \& McDiarmid, R. W. 1999. Body plan: development and morphology. In: McDiarmid, R. W. \& Altig, R. eds. Tadpoles: the biology of anuran larvae. Chicago, The University of Chicago Press. p. 24-51.

De SÁ, R. O.; Wassersug, R. \& KeHr, A. I. 1997. Description of tadpoles of three species of Scinax (Anura: Hylidae). Herpetological Journal 7:13-17.

Duellman, W. E. 1970. The hylid frogs of Middle America. Museum of Natural History of the University of Kansas Monograph 1:1-753. . 1978. The biology of an equatorial herpetofauna in Amazonian Ecuador. Museum of Natural History of the University of Kansas Miscellaneous Publications 65:1-352.

. 2005. Cusco Amazónico. The lives of amphibians and reptiles in an Amazonian rainforest. Ithaca, London, Comstock Publishing Association. 433p.

Duellman, W. E. \& Trueb, L. 1986. Biology of Amphibians. McGrawHill, New York. 670p.

. 1994. Biology of Amphibians. Baltimore, The Johns Hopkins University Press. 670p.

Duellman, W. E. \& Wiens, J. J. 1992. The status of the hylid frog genus Ololygon and the recognition of Scinax Wagler, 1830. Occasional Papers of the Museum of Natural History, University of Kansas 151:1-23.

Faivovich, J. 2002. A cladistic analysis of Scinax (Anura: Hylidae). Cladistics 18(4):367-393. 
Faivovich, J.; Haddad, C. F. B.; Garcia, P. C. A.; Frost, D. R.; Campbell, J. A. \& WheELER, W. C. 2005. Systematic review of the frog family Hylidae, with special reference to Hylinae: a phylogenetic analysis and taxonomic revision. Bulletin of the American Museum of Natural History 294:1-240.

Frost, D. R. 2013. Amphibian Species of the world: an Online Reference. Version 5.5. New York, American Museum of Natural History. Disponível em: <http://research.amnh.org/vz/herpetology/ amphibia/>. Acessado em 18.03.2013.

GosNer, K. L. 1960. A simplified table for staging anuran embryos and larvae wit notes on identification. Herpetologica 16:183-190.

Hero, J. M. \& Mijares-Urrutia, A. 1995. The tadpole of Scinax rostrata (Anura: Hylidae). Journal of Herpetology 29(2):307-311.

Hoogmoed, M. S. \& Gruber, U. 1983. Spix and Wagler type specimens of reptiles and amphibians in the Natural History Museum in Munich (Germany) and Leiden (The Netherlands). Spixiana, Supplement 9:319-415

LEÓN, J. R. 1969. The systematics of the frogs of the Hyla rubra group in Middle America. University of Kansas Publications, Museum of Natural History 18(6):505-545.
Lynch, J. D. 2006. The tadpoles of frogs and toads found in the lowlands of northern Colombia. Revista de la Academia Colombiana de Ciencias 30(116):443-457.

McDiarmid, R. W. \& Altig, R. 1990. Description of a bufonid and two hylid tadpoles from western Ecuador. Alytes 8(2):51-60.

1999. Research: materials and techniques. In: McDiarmid, R. W.

\& Altig, R. eds. Tadpoles: the biology of anuran larvae. Chicago, The University of Chicago Press. p. 7-23.

Rada De Martinez, D. 1990. Contribución al conocimiento de las larvas de anfibios de Venezuela. Memória de la Sociedad de Ciencias Naturales La Salle 49/50:391-403.

Sturaro, M. J.; Sarmento, J. F. M.; Lima, A. A.; Chalkidis, H. M. \& Rocha, R. A. T. 2010. New records and distribution of the treefrog Scinax rostratus (Peters, 1863) (Amphibia: Anura: Hylidae). Herpetology Notes 3:161-166. 